\title{
Modelo para el Control de Inundaciones durante el Fenómeno De "La Niña" Utilizando un Embalse Hidroeléctrico
}

\author{
Victor H. Bedoya ${ }^{(1)}$ y Jesús M. López-Lezama ${ }^{(2)}$ \\ (1) XM S.A E.S.P, Calle 12 Sur No. 18-168, Medellín - Colombia (e-mail vbedoyar@xm.com). \\ (2) Grupo de Investigación GIMEL, Departamento de Ingeniería Eléctrica, Facultad de Ingeniería, \\ Universidad de Antioquia, Calle 70 No.52-21, Medellín - Colombia (e-mail: lezama@udea.edu.co).
}

Recibido Jul. 1, 2014; Aceptado Sep. 16, 2014; Versión final recibida Oct. 9, 2014

\begin{abstract}
Resumen
En este artículo se presenta un modelo para control de inundaciones durante la temporada de lluvias y en especial durante el fenómeno de "La Niña". El modelo propuesto optimiza el recurso hídrico de una planta de generación hidroeléctrica maximizando los ingresos por venta de energía del generador, pero considerando restricciones y penalizaciones por control de inundaciones. La principal contribución del artículo es la inclusión de criterios de responsabilidad social en un esquema de despacho energético. El modelo es probado en un sistema real, en una zona geográfica susceptible a inundaciones regularmente. El esquema de despacho energético resultante garantiza confiabilidad en la zona de control de inundaciones de acuerdo a las restricciones planteadas.
\end{abstract}

\section{Flood Control Model during "La Niña” Phenomenon using a Hydroelectric Reservoir}

\begin{abstract}
This paper presents a model for flood control during rainy seasons, and especially during the phenomenon of "La Niña". The proposed model optimizes the hydric resources of a hydroelectric plant maximizing the income of the generator for energy sales but considering constraints and penalizations for flood control. The main contribution of the paper is the inclusion of social responsibility criteria within an energetic economic dispatch. The proposed model is tested in a real system in a geographic area where floods occur regularly. The resulting energetic dispatch scheme guarantees reliability in the flood control zone according to the established constraints.
\end{abstract}

Keywords: flood control, multipurpose reservoir, phenomenon of La Niña, flood modeling 


\section{INTRODUCCIÓN}

El cambio climático a nivel mundial y los eventos climáticos extremos plantean nuevos retos en la planeación de los recursos hidráulicos y la gestión de riesgos de afectación social. Eventos como las inundaciones presentan una afectación directa e indirecta sobre la población; y por tanto, incentivan a nuevas propuestas para la administración de la infraestructura existente y/o futura designada para el control y aprovechamiento del recurso hídrico (Minville et al., 2010). En particular, la planeación y operación de los proyectos hidroeléctricos puede ser orientada de tal forma, que busque minimizar el impacto negativo en la en la zona influencia debido a inundaciones.

En la zona ecuatorial del continente americano se producen variaciones climáticas que no obedecen a los mismos procesos observados en las altas latitudes responsables de las estaciones, debido a que el clima de esta zona permanece relativamente estable durante todo el año (Leiva et al., 2001). Por tanto, el clima es afectado por ligeras variaciones que permiten identificar dos periodos climáticos conocidos como temporada seca y temporada de lluvias. Debido a la alteración de las condiciones climáticas, se presentan fenómenos como "El Niño" y "La Niña", los cuales se caracterizan por déficit y exceso de lluvias, respectivamente. Estos fenómenos son complementarios y constituyen el ciclo de oscilación del sur; no obstante, la característica cíclica no puede ser reflejada con una periodicidad de ocurrencia fija debido a su alta variabilidad, lo cual complica la predicción de estos fenómenos y la preparación logística ante sus consecuencias (Philander et al., 1995).

Los eventos de inundaciones tienen presencia a nivel mundial y generalmente tienen una mayor concentración en zonas costeras y cercanas a las riveras de los ríos (Field et. al., 2012), la temporada de lluvias y en especial el fenómeno de "La Niña" imponen escenarios de aportes hídricos altos, que facilitan la ocurrencia de inundaciones y sus consecuencias en la población, infraestructura y economía de la zona afectada. En países que se caracterizan por el aprovechamiento del recurso hídrico para la producción de energía eléctrica, se cuenta con infraestructura dispuesta para embalsamiento. Un embalse puede presentar dentro de sus funciones una característica de operación unipropósito o multipropósito dependiendo de la realización de una o varias de las siguientes actividades: generación de energía eléctrica, suplencia de acueductos, control de inundaciones, control del afluente para su navegación, fines recreativos y control de flujo para garantizar aspectos ecológicos en la zona de influencia (Xu \& Zhao, 2010; Hao, 2009). El número de propósitos de cada embalse es dependiente del objetivo planteado para la realización del proyecto, ya que el embalsamiento de agua a gran escala, generalmente representa un alto costo de inversión y, en ocasiones, es dependiente de una tasa de retorno atractiva.

La infraestructura conocida como unipropósito, designada para la generación de energía eléctrica y ubicada aguas arriba de un área con riesgo de inundación, puede ser administrada para realizar un control de inundaciones efectivo con el fin de mitigar o evitar una posible inundación. Un modelo energético centralizado con el objetivo de coordinar el despacho de las plantas hidráulicas en una región, ayudaría a mitigar los efectos negativos en la población durante la temporada de lluvias y en especial durante un fenómeno de la Niña. Los modelos energéticos tradicionales de despacho de potencia eléctrica como los presentados en (Jothiprakash et al., 2011)(Ma et al., 2011)(Villada et al., 2011) (Catalao et al., 2007) y (Musgens, 2006) no contemplan el control de inundaciones ni modelan esfuerzos realizados de manera conjunta por el operador del sistema eléctrico y los agentes administradores de los embalses hidroeléctricos, para mitigar inundaciones aguas abajo de los embalses, siendo dominados por el criterio económico que rige los mercados eléctricos.

En las últimas cinco décadas, los eventos de inundaciones han sido responsables de más de la mitad del costo total de los desastres naturales en el mundo. Un ejercicio de control de inundaciones en las represas de Longyangxia y Liujiaxia en China propone compensaciones económicas por control de inundaciones por tramos del río afluente (Xu \& Zhao, 2010). En Vietnam se propuso para el río Rojo, la operación de un

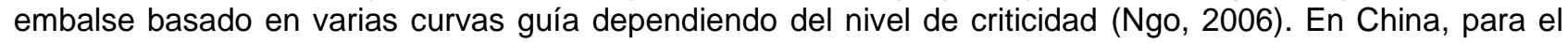
proyecto de Tres Gargantas, se propuso un modelo de aplicación solo durante la estación de lluvias, aplicando un análisis de confiabilidad y riesgo basado en los pronósticos hidrológicos (Dong et al., 2010). En Malaysia se propuso la operación de embalses en cascada, coordinada por medio de curvas guía, en el cual los volúmenes de los embalses se encuentran relacionados por curvas (Asfaw et al. 2011). En China se propuso un esquema de operación de embalses para el control de inundaciones basado en información en tiempo real y pronósticos (Guohua et al. 2009). En Mozambique se planteó el control de inundaciones utilizando un embalse hidroeléctrico para mitigar el impacto social y reflejando la afectación sobre la generación de energía eléctrica (Beilfuss, 2010). En Canadá se trató el tema de control de inundaciones aguas arriba y abajo de un embalse considerando la afectación de la cuenca aguas abajo de la planta, analizando las descargas de agua producidas por la planta y de acuerdo a las estaciones climatológicas (Fortier et al. 2011). 
En este artículo se propone un modelo estocástico para un sistema energético de una planta hidroeléctrica, con el fin de utilizar su embalse como elemento primario para el control de inundaciones durante la temporada de lluvias y en especial el fenómeno de la Niña. El control de inundaciones se propone en un punto ubicado aguas abajo de la planta. El modelo energético incluye criterios de responsabilidad social por control de inundaciones, los cuales permiten simular el impacto sobre la generación de energía de la planta y la confiabilidad en el punto de control de inundaciones.

\section{DESCRIPCIÓN DEL CASO DE ESTUDIO}

El modelo básico del sistema energético de una planta hidroeléctrica está compuesto por un embalse, sus flujos de entrada y salida. En la Fig. 1 se ilustra el sistema bajo análisis. A partir del modelo básico, se presentan las siguientes variables: flujo aportado por la cuenca aguas arriba del embalse (Q1), flujo de descarga del embalse con fines de producción de energía eléctrica (Q3), flujo de descarga por vertimiento (Q2), flujo aportado por la cuenca aguas abajo del embalse entre el punto de descarga y el punto de control de inundación (Q5), un flujo atribuible a un río externo, el cual no es controlado por el embalse en estudio (Q4), un flujo de desbalance del sistema propio del embalse (evaporación + filtración) y por último una zona de control de inundaciones, en la cual el flujo (Q6) es controlado con el fin de proteger una población. En la Tabla 1 se presentan los parámetros de la planta hidroeléctrica en estudio, los cuales pertenecen a una planta real ubicada en el norte de Colombia.

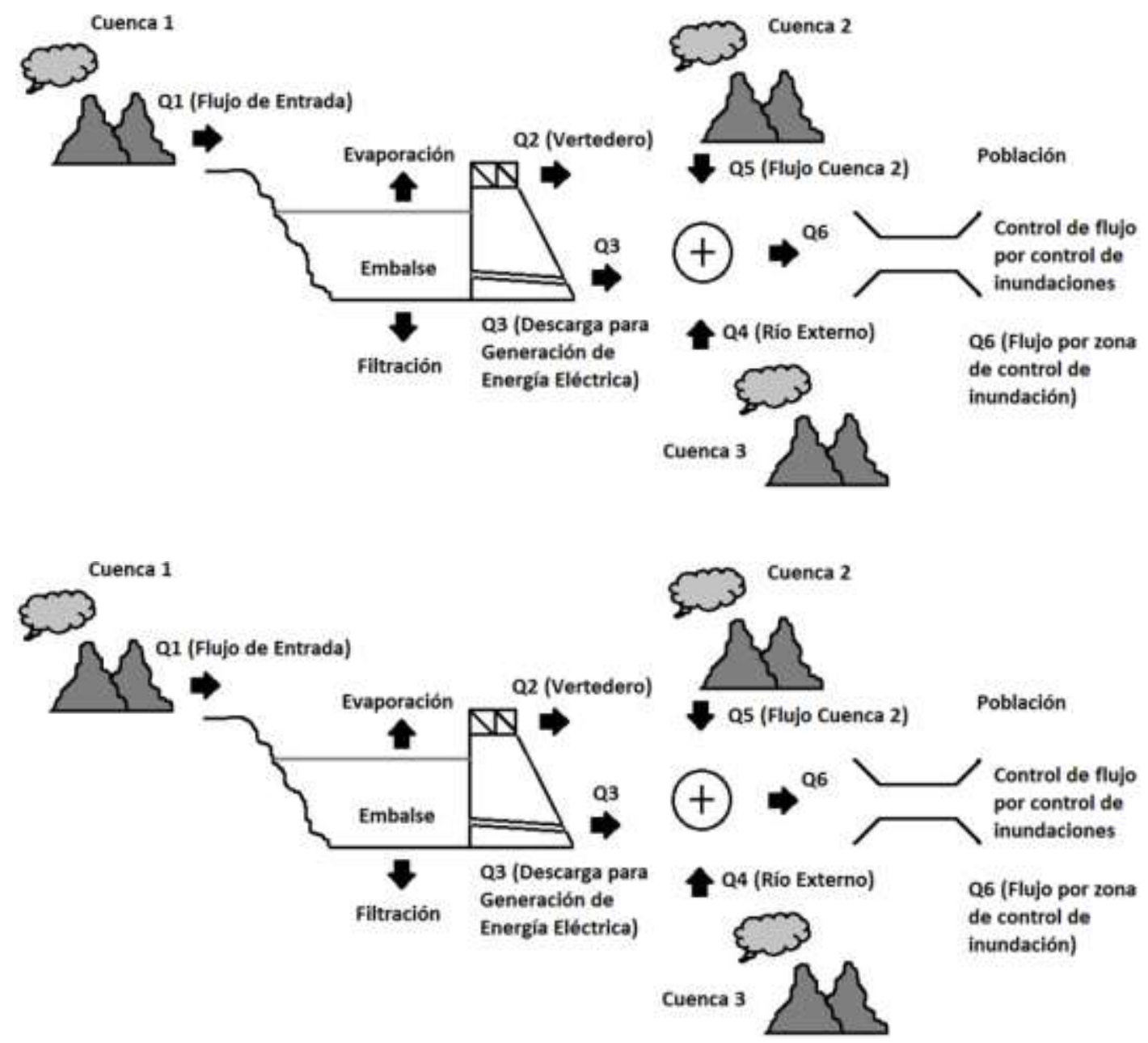

Fig.1: Diagrama de Flujos en el modelo propuesto

Para el caso de estudio, las variables Q1 y Q4 son modeladas como series hidrológicas. El flujo hallado en Q5 es dependiente del flujo Q1 ya que se supone una misma cuenca, separada por el dique del embalse. Este procedimiento se explica en la Fig.3. Los valores de evaporación y filtración son dependientes del área superficial y el volumen del embalse, respectivamente. El valor de la evaporación fue considerado con un potencial anual promedio de $1826 \mathrm{~mm}$ (Ruiz et al., 2008), lo que significa un valor promedio de $5 \mathrm{~mm} /$ día. Para el valor de filtración se utilizó una constante típica para suelos moderadamente permeables de $1 \times 10^{-3}$ cm/s (Terzaghi et. al.,1980). Los Flujos Q3 y Q6 son resultados del modelo de simulación propuesto. 
Tabla 1: Parámetros de la planta hidroeléctrica en estudio

\begin{tabular}{|l|c|}
\hline Descripción & Valor \\
\hline Volumen Máximo del Embalse [Mm3] & 1863,54 \\
\hline Volumen Mínimo técnico [Mm3] & 358,97 \\
\hline $\begin{array}{l}\text { Volumen Máximo de descarga por conducto dedicado a generación de potencia } \\
\text { eléctrica por un día [Mm3] }\end{array}$ & 60,48 \\
\hline $\begin{array}{l}\text { Volumen Mínimo de descarga por conducto dedicado a generación de potencia } \\
\text { eléctrica por un día [Mm3] }\end{array}$ & 6,48 \\
\hline Nivel máximo del embalse [m.s.n.m] & 130,5 \\
\hline
\end{tabular}

En la Fig. 2 se ilustran las curvas guía que delimitan la región de operación del embalse presentado en el caso de estudio y parametrizado según la Tabla 1. En la Fig.2 la curva guía mínima cumple con obligaciones en la operación del embalse correspondiente a temas ambientales tales como: caudal y niveles del río necesarios para conservar niveles seguros de oxígeno, la calidad del agua, simulación de niveles históricos del río, mínima descarga que se debe mantener todo el tiempo, mantener los niveles históricos del río para permitir cambios en el ecosistema, suplir acueductos y mantener la navegabilidad del río. La curva guía máxima es la responsable del control de crecientes para evitar vertimientos y pérdida de control del flujo liberado aguas abajo (Asfaw et al., 2011).

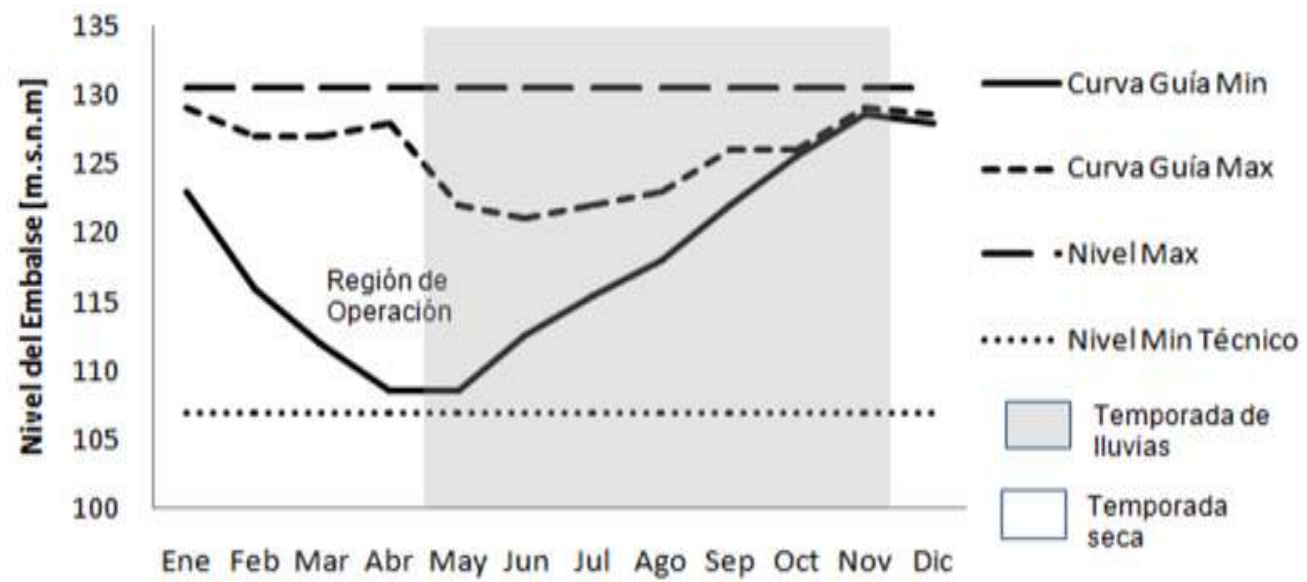

Fig.2: Curva guía de operación del embalse

Las cuencas presentadas en las figuras 1 y 3 como Cuenca 1 y Cuenca 2 son fraccionadas acorde a la frontera común que es el dique del embalse o represa. Esta discriminación se realiza con el fin de separar los aportes de la cuenca aguas arriba y aguas abajo del embalse. Una cuenca hidrográfica está definida como el límite geográfico donde los aportes por lluvia se consideran como una contribución al río principal o finalmente al embalse o punto de control (Mahmud \& Hashim, 2011). Para conocer el aporte de la cuenca 2 (Q5) al punto de control ubicado en la población aguas abajo, se utiliza la ecuación (1) que define el rendimiento para una cuenca.

$\mathrm{R}_{\text {cuenca }}=\frac{\mathrm{Q}}{\mathrm{A}}\left[\frac{\frac{1}{\mathrm{~s}}}{\mathrm{Km}^{2}}\right]$

Donde $R_{\text {cuenca }}$ es el rendimiento de la cuenca, $Q$ es el flujo de entrada al embalse medido o estimado en la región de estudio y $A$ es el área de la cuenca donde la contribución por lluvia se refleja en una contribución sobre la región de estudio. Un cálculo simplificado de la contribución de la cuenca 2 sobre el punto de control de inundaciones puede ser llevado a cabo bajo las siguientes consideraciones: i) Las cuencas Cuenca 1 y Cuenca 2 poseen un suelo homogéneo; ii) Las condiciones del clima en Cuenca 1 y Cuenca 2son homogéneas; y iii) Área 1 >> Área2. Cumpliendo con las consideraciones anteriores, se puede inferir que se cumple con la ecuación (2).

$\mathrm{R}_{\text {cuenca } 1}=\mathrm{R}_{\text {cuenca } 2}$

A partir de las ecuaciones (1) y (2) es posible calcular la contribución de la cuenca 2 (Q5) en la zona de control de inundaciones ubicada aguas abajo del embalse. 


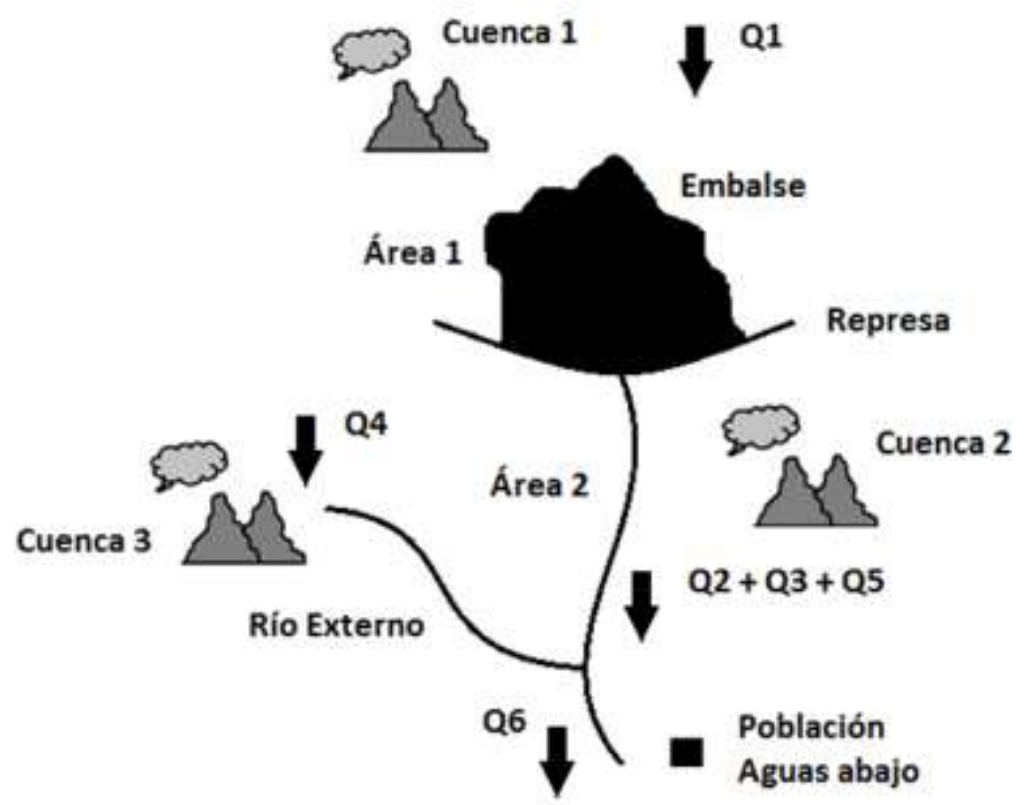

Fig.3: Diagrama de cuenca del caso de estudio

\section{METODOLOGÍA}

El modelo de simulación propuesto fue planteado mediante la utilización del método de Monte Carlo sobre un modelo de optimización estocástica implícita y fue desarrollado en Optimization Programming Language $(\mathrm{OPL})$. El objetivo del modelo es encontrar una programación óptima de despachos de generación con el fin de maximizar los ingresos económicos de la planta hidroeléctrica por venta de energía, mientras se satisfacen diferentes restricciones por control de inundaciones en términos de confiabilidad. En la Fig. 4 se ilustra el modelo de simulación propuesto para intentar anticipar una señal de operación sobre un embalse hidroeléctrico. El corazón del modelo estocástico es un modelo de optimización determinístico, el cuál puede ser desarrollado bajo cualquier técnica de optimización y comprende un modelo para un escenario hidrológico único.

En un modelo energético, la predicción hidrológica conlleva un nivel alto de incertidumbre al intentar recrear un escenario hidrológico futuro. Por medio de la estocasticidad se abarca un gran número de escenarios posibles, con el fin de establecer probabilidades de ocurrencia, y acorde a los resultados, tomar decisiones bajo niveles de confianza. El modelo de optimización estocástico propuesto requiere de los siguientes insumos: i) Series hidrológicas: ii) Modelo de optimización determinístico; iii) Restricciones planteadas al modelo determinístico; y iv) Estudio de confiabilidad aplicado a la zona de control de inundaciones.

i) Series hidrológicas: A través de un generador de series sintéticas y un pronóstico hidrológico, se construye la cantidad de series hidrológicas deseada según la resolución de tiempo que se plantee en el modelo de optimización. Se debe tener en cuenta una distribución probabilística dependiente de los datos históricos reales para cada flujo y el pronóstico del mismo. Mediante el generador de series sintéticas se haya las series de los flujos Q1 y Q4.

ii) Modelo de optimización determinístico: El modelo fue desarrollado bajo características lineales y es el encargado de encontrar el despacho óptimo de generación maximizando el ingreso económico de la planta hidroeléctrica por venta de energía, mientras se satisfacen las diferentes restricciones por control de inundaciones para cada combinación de series hidrológicas.

iii) Restricciones planteadas al modelo determinístico: El modelo determinístico posee una formulación matemática lineal y sus restricciones son lineales, las restricciones planteadas proponen penalizaciones sobre la función objetivo por inundaciones en la zona de control, penalizaciones por vertimiento y penalizaciones por situar el punto de operación del embalse por fuera de la región delimitada por las curvas guía.

iv) Estudio de confiabilidad aplicado a la zona de control de inundaciones: Finalizado el proceso iterativo para cada una de las series hidrológicas consideradas en el estudio, se cuenta con un número de despachos hidráulicos igual al número de series planteado, con esta cantidad de despachos se evalúa el impacto generado en la zona de control de inundación y se establece el nivel de confiabilidad en la zona. 
Aplicando el método de Monte Carlo, se soluciona el sistema determinístico para cada combinación de series los flujos Q1 y Q4 acorde al número de combinaciones deseado.

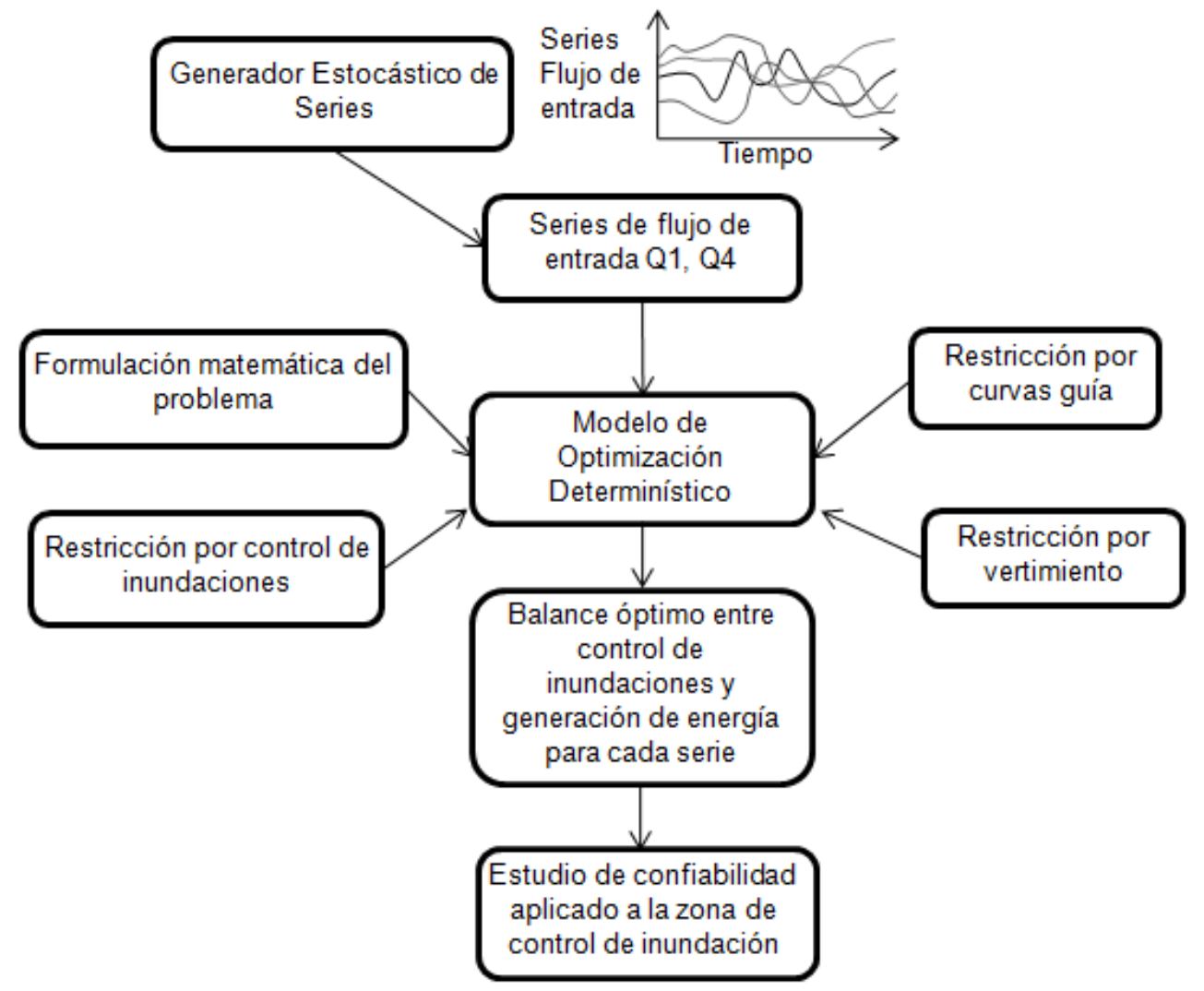

Fig.4: Estructura del modelo de optimización estocástico propuesto

\section{Función objetivo}

La función objetivo está dada por la ecuación (3) y las restricciones se encuentran plasmadas en las ecuaciones (4) a (7). La función objetivo contiene penalizaciones por vertimiento, inundaciones en el punto de control ubicado aguas abajo del embalse y operación del embalse por fuera de la región delimitada por las curvas guía.

\begin{tabular}{|c|c|}
\hline $\operatorname{Max} \sum_{i=1}^{N_{-} \text {dias }}\left(\begin{array}{c}C_{i} * C F_{i} * V Q_{3_{i}}-V Q_{2_{i}} * \text { Pen }_{\text {vertimiento }} \\
-\operatorname{Vol}_{\text {desv-curva }_{i}} * \text { pen }_{\text {des-curva }}-\text { Vol }_{\text {inundación }} * \text { pen }_{\text {inundación }}\end{array}\right)$ & (3) \\
\hline$V Q_{3 \min } \leq V Q_{3_{i}} \leq V Q_{3 \max }$ & (4) \\
\hline$V_{\text {min_técnico }} \leq V_{i} \leq V_{\max }$ & $(5)$ \\
\hline$Q_{6}=Q_{2}+Q_{3}+Q_{4}+Q_{5}$ & (6) \\
\hline $\mathrm{Q}_{6} \leq \mathrm{Q}_{6_{\max }}$ & (7) \\
\hline$V_{i}=V_{0_{i}}+V Q_{1_{i}}-V Q_{2_{i}}-V Q_{3_{i}}-V_{\text {Evaporación }+ \text { filtración }_{i}}$ & (8) \\
\hline
\end{tabular}

El flujo por la zona de control de inundación se encuentra definido en la ecuación (6). La ecuación (7) contiene el límite seguro para evitar inundaciones en la zona de control. La ecuación (8) contiene el balance de masa propio del embalse, partiendo del principio de que lo que entra al embalse debe ser igual a lo que sale, teniendo en cuenta la capacidad de almacenamiento del sistema. La Tabla 2 presenta la descripción de las variables contenidas en la función objetivo y restricciones del problema. 
Tabla 2: Descripción de variables contenidas en función objetivo y restricciones

\begin{tabular}{|c|c|c|}
\hline Variable & Unidades & Descripción \\
\hline$i$ & Días & Periodo de tiempo \\
\hline$C_{i}$ & COP/GWhD & Costo de la energía promedio para el periodo i \\
\hline$C F_{i}$ & $\mathrm{GWhD} / \mathrm{Mm}^{3}$ & Factor de conversión en el periodo i \\
\hline$V_{o_{i}}$ & $\mathrm{Mm}^{3}$ & Volumen inicial del embalse en el periodo i \\
\hline$V Q_{3_{i}}$ & $\mathrm{Mm}^{3}$ & $\begin{array}{l}\text { Volumen de descarga del embalse utilizando los ductos para } \\
\text { generación de Energía Eléctrica en el periodo i }\end{array}$ \\
\hline$V Q_{2_{i}}$ & $\mathrm{Mm}^{3}$ & Volumen de descarga utilizando el vertedero en el periodo i \\
\hline$V Q_{1_{i}}$ & $\mathrm{Mm}^{3}$ & $\begin{array}{l}\text { Volumen aportado por la cuenca aguas arriba del embalse en el } \\
\text { periodo i }\end{array}$ \\
\hline Pen $_{\text {vertimiento }}$ & $\mathrm{COP} / \mathrm{Mm}^{3}$ & Penalización por vertimiento de agua \\
\hline Vol $_{\text {desv-curva }}$ & $\mathrm{Mm}^{3}$ & $\begin{array}{l}\text { Volumen diferencia entre el punto de operación del embalse y la } \\
\text { región delimitada por las curvas guía en el periodo i }\end{array}$ \\
\hline pen $_{\text {desv-curva }}$ & $\mathrm{COP} / \mathrm{Mm}^{3}$ & $\begin{array}{l}\text { Penalización por cada } \mathrm{Mm}^{3} \text { por fuera de la región delimitada por } \\
\text { las curvas guía }\end{array}$ \\
\hline Volinundación $_{i}$ & $\mathrm{Mm}^{3}$ & $\begin{array}{l}\text { Volumen diferencia entre el volumen en la zona de control de } \\
\text { inundación y el límite seguro en el periodo i }\end{array}$ \\
\hline pen $_{\text {Inundación }}$ & $\mathrm{COP} / \mathrm{Mm}^{3}$ & $\begin{array}{l}\text { Penalización por cada } \mathrm{Mm}^{3} \text { por encima del límite seguro en la zona } \\
\text { de control de inundación }\end{array}$ \\
\hline$V_{i}$ & $\mathrm{Mm}^{3}$ & Volumen del embalse en el periodo i \\
\hline$V_{\text {Evaporación }+ \text { filtración }}$ & $\mathrm{Mm}^{3}$ & Volumen de evaporación + Volumen de filtración en el periodo i \\
\hline
\end{tabular}

\section{Series de flujo Q1 YQ4}

Para la construcción de las series Q1 y Q4, se requiere cada uno de los pronósticos calculados para los respectivos caudales. A partir de los pronósticos, el generador de series de caudales construye la cantidad de series requeridas acorde a una distribución probabilística normal, cuya media es el valor del pronóstico para cada flujo y la desviación estándar es dependiente de los valores históricos respectivos. El caso de estudio abarca un periodo de tiempo de 31 días. La base de datos comprendida para los datos históricos es de 15 años en los cuales se ha presentado al menos un fenómeno de la "Niña". En la Fig. 5 se ilustra la franja de los valores de los caudales Q1 y Q4 obtenidos a partir del generador de series de caudales.
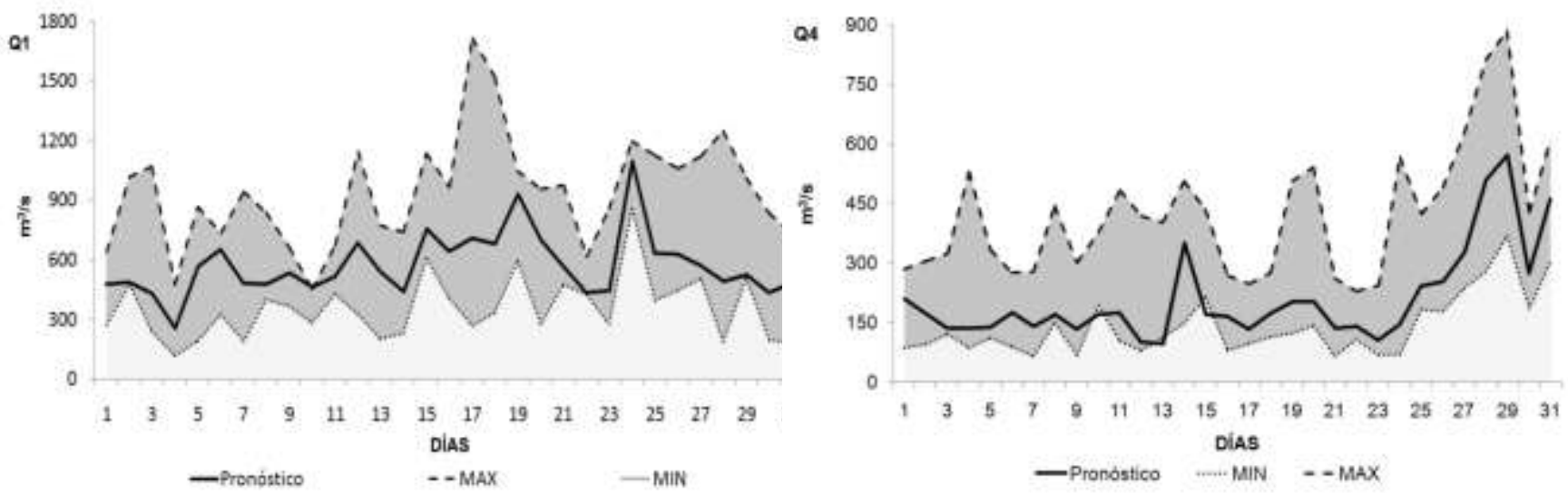

Fig.5: Series de caudales propuesta para los flujos Q1 y Q4

En la Fig.5 se presentan los pronósticos de los caudales para los flujos Q1 y Q4, la franja delimitada por los valores MAX y MIN muestra los valores extremos de las series de caudal reproducidas por el generador de series de caudales, es decir, el número de series generadas para el estudio se encuentra contenida en cada una de las franjas respectivamente. 


\section{RESULTADOS}

En Fig.6, Fig.7 y Fig.8 se ilustran los resultados obtenidos para el cálculo del flujo Q6 correspondiente al flujo que transita por la zona de control de inundación. La Fig. 6 muestra el caso de estudio aplicando a la función objetivo únicamente la penalización por vertimiento. La Fig. 7 presenta el caso de estudio aplicando a la función objetivo las penalizaciones por vertimiento y operación del embalse por fuera de las curvas guía.

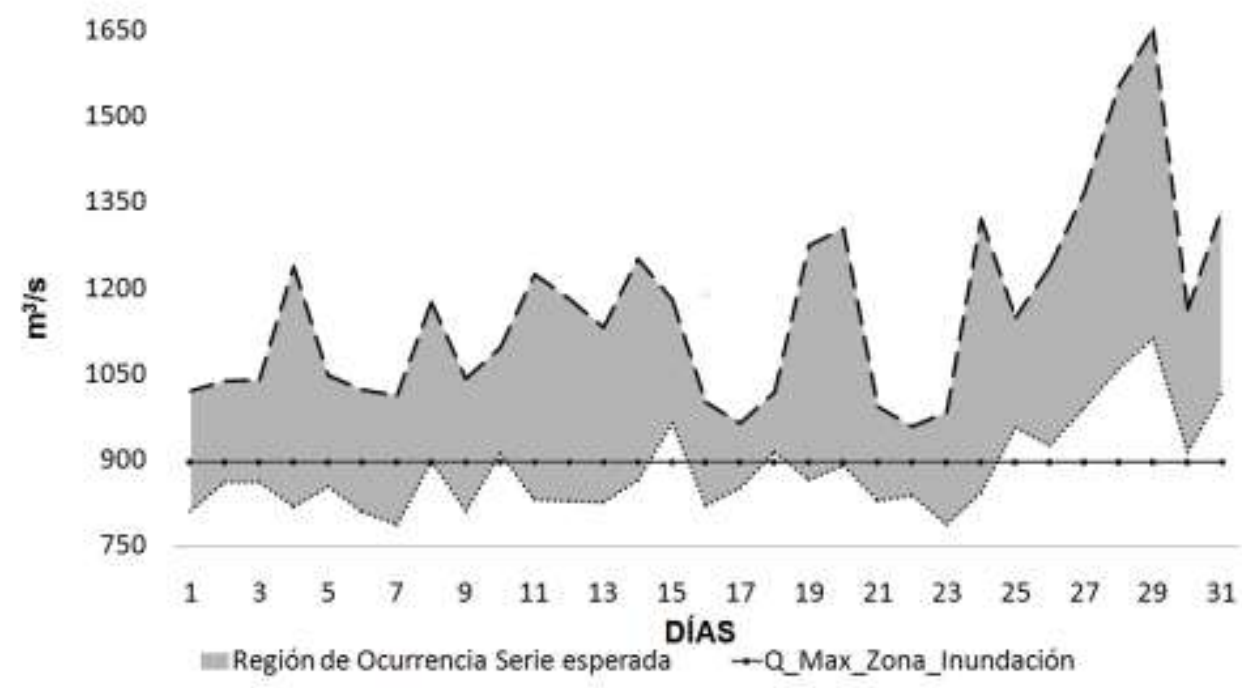

Fig.6: Caso de estudio solo con penalización por vertimiento

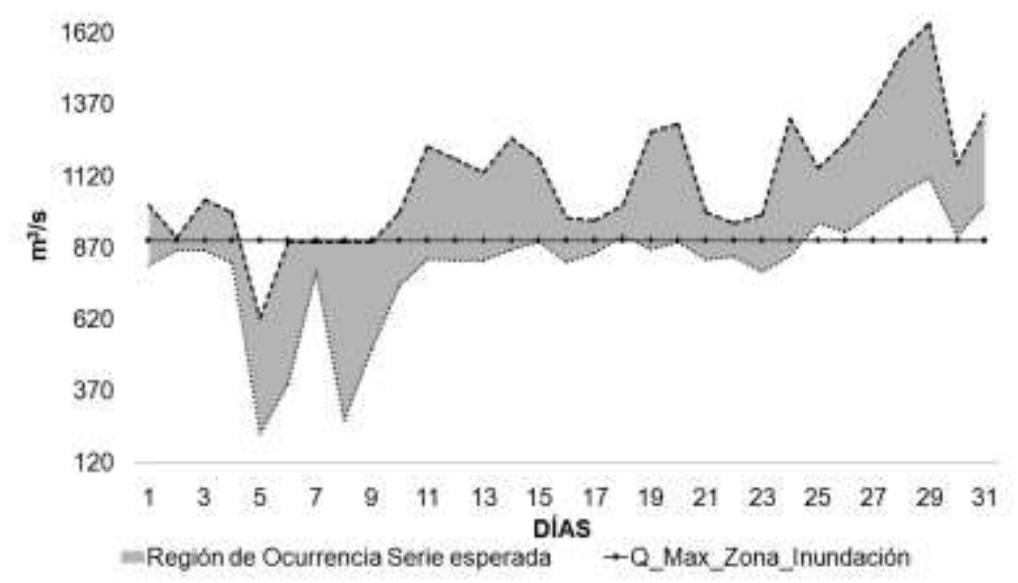

Fig.7: Caso de estudio solo con penalización por vertimiento y punto de operación del embalse por fuera de la región delimitada por las curvas guía

En la Fig. 6 y Fig. 7 predomina la característica presentada por las series obtenidas para el flujo Q4, es decir, para lograr un control de inundaciones en la zona de control, el embalse debe estar dispuesto a operar en función del flujo Q4. La Fig.6 muestra que un embalse sin la utilización de curvas guía para su operación y sin información sobre los flujos aguas abajo de la planta, es un embalse que puede causar una gran afectación por inundaciones sobre la población ubicada aguas abajo. La Fig. 7 muestra la cantidad de flujo por encima del valor máximo permitido para el flujo Q6 con el fin de evitar inundaciones. Para este caso, el embalse opera con penalizaciones en la función objetivo por curvas guía y vertimientos. Los resultados reflejan que las curvas guía ofrecen una señal directa para el control de crecientes, pero si no se cuenta con el debido monitoreo de zona de afectación, el sistema no cumple satisfactoriamente con el objetivo de mitigar el impacto por inundaciones en la zona de control. La Fig. 8 ilustra el caso de estudio aplicando penalizaciones a la función objetivo por vertimiento, operación del embalse por fuera de la región delimitada por las curvas guía e inundaciones en la zona de control de inundación. 
La Fig. 8 presenta el caso en el cual el sistema opera de manera conjunta con todas las penalizaciones. Para este caso el modelo logra establecer que se maximice la generación de la planta de manera segura para la zona de control de inundaciones, situándose la mayor parte del tiempo en el límite seguro de la zona. Una operación de este estilo puede ser considerada como riesgosa, en este caso el límite seguro debe ser confiable con el fin de contar con margen de respaldo. La Fig. 9 muestra la confiabilidad en la zona de control de inundaciones para los casos expuestos en Fig.6, Fig.7 y Fig.8.

En Fig. 9 se puede observar que la confiabilidad más baja sobre la zona de control de inundaciones se obtiene cuando solo se presenta penalización en la función objetivo por vertimiento, la confiabilidad aumenta con el caso de las penalizaciones para operaciones del embalse por fuera de las curvas guía, sin embargo, la confiabilidad óptima se consigue con el control de inundaciones con información directamente del sitio a controlar.

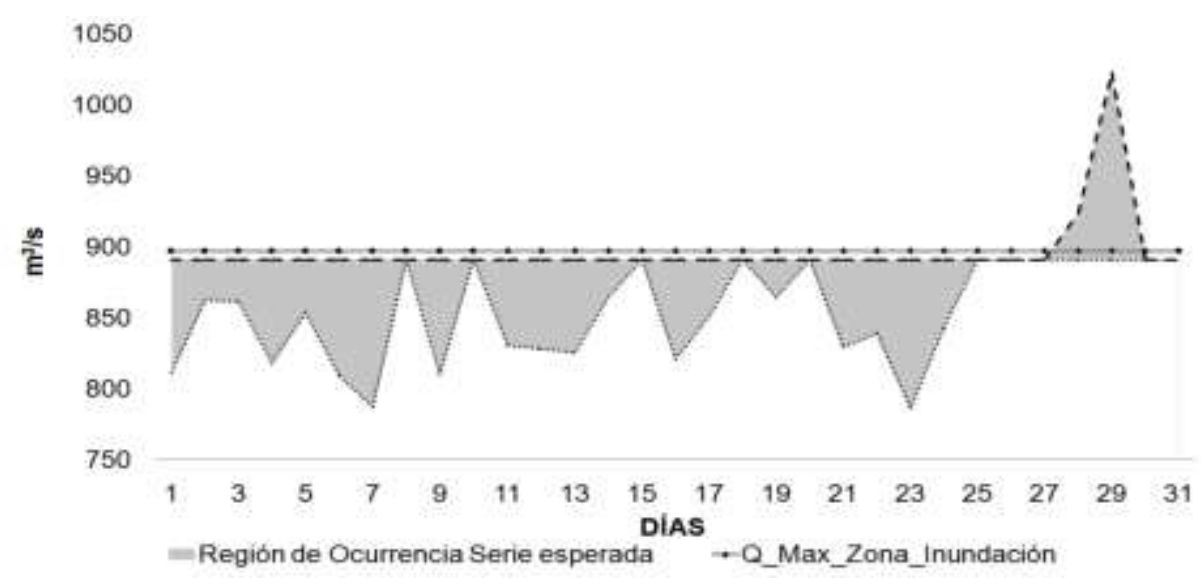

Fig.8: Caso de estudio con penalizaciones por vertimiento, operación por fuera de la región delimitada por las curvas guía y por inundación en la zona de control.

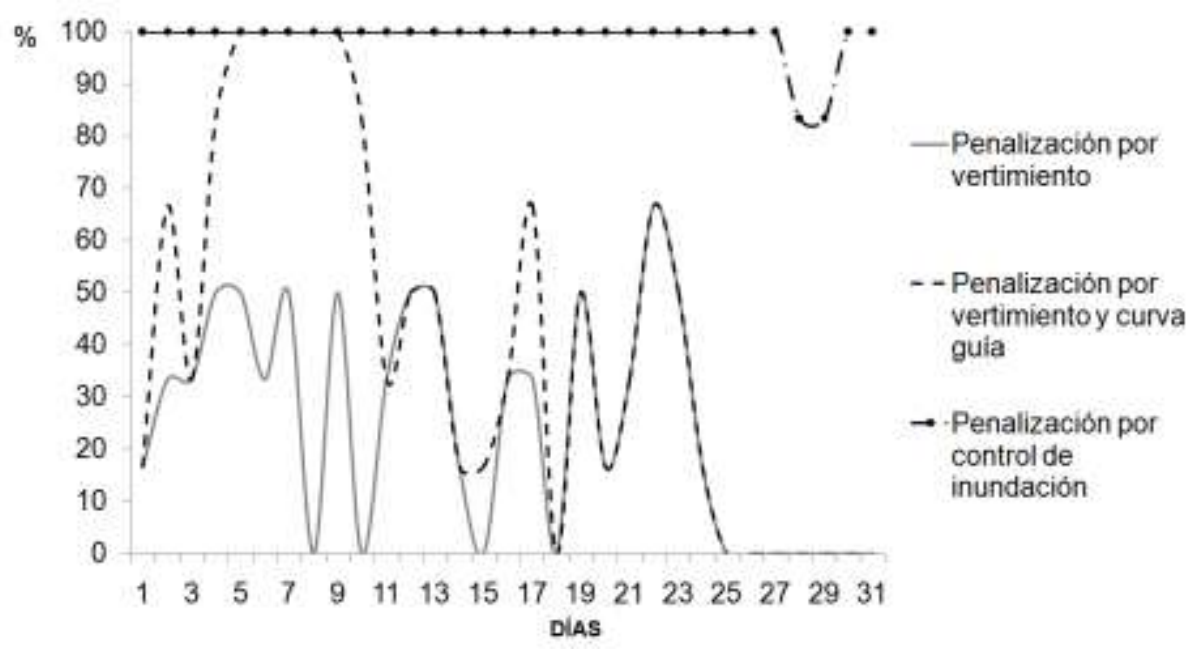

Fig.9: Confiabilidad en zona de control de inundaciones

\section{CONCLUSIONES}

El modelo analizado en este artículo arroja una señal sobre cambiar el propósito de los embalses hidroeléctricos durante la temporada de lluvias y en especial durante el fenómeno de "La Niña", con el fin de garantizar el control de inundaciones en la población. Dos objetivos deben ser fundamentales: el primero es evitar perder el control de crecientes por parte del embalse (curvas guía) y el segundo es evitar inundar en la zona de control de inundación.

El control de inundaciones con señales referenciadas directamente en el punto de control, se convierte en una señal muy efectiva al momento de coordinar las descargas del embalse; pero es claro que puede ocasionar fluctuaciones en los despachos de generación por parte de la planta hidráulica. Esta situación 
impacta la remuneración de los generadores por venta de energía, añadiendo un reto adicional sobre incentivos económicos a los agentes operadores de embalses hidroeléctricos en el país.

Las ecuaciones lineales planteadas para el control de inundaciones en una zona de control, propuestas bajo penalizaciones a la función objetivo, reflejan una realidad para un sistema inmerso en un mercado de energía en el cual se busca la maximización de utilidades bajo el cumplimiento de normas regulatorias y satisfacción de restricciones de actividades multipropósito.

La confiabilidad en la zona de control de inundaciones aumenta significativamente cuando se monitorea de forma puntual, es por esta razón que la mezcla entre curvas guía y monitoreo de la zona de control para la toma de decisiones en la administración del embalse, muestra una buena práctica para la futura coordinación de sistemas de embalses sobre una zona en común.

\section{AGRADECIMIENTOS}

Los autores agradecen a la empresa XM S.A. E.S.P y a la Universidad de Antioquia (UdeA) por el apoyo del proyecto "Sostenibilidad 2014-2015" para el desarrollo de este trabajo.

\section{REFERENCIAS}

Asfaw, T. D., Yusof, K. W., \& Hashim, A. M., Parameters estimation and rule curve development of cascade hydropower reservoirs. Proceedings of the National Postgraduate Conference (NPC), 1-5, Kuala Lumpur, 19 a 20 de Septiembre (2011).

Beilfuss, R., Modelling trade-offs between hydropower generation and environmental flow scenarios: A case study of the Lower Zambezi River Basin, Mozambique. International Journal of River Basin Management 8(1), 331-347 (2010).

Catalao J.P., Mariano S.J., Mendes V.M., y Ferreira L.A., Planificación de la operación de corto plazo de sistemas de energía termoeléctrica con restricciones ambientales, Revista Inf. Tecnol. Cit: 18(1), 79-86, (2010).

Dong, X., Liu, J., Li, Y., Bo, H., \& Deng, X., Dynamic Application and Risk Analysis of Flood Control Water Level to the Three Gorges Reservoir by Utilizing Mid-Term Inflow Forecasts. Proceedings of the IEEE AsiaPacific Power and Energy Engineering Conference, 1-5, Chengdu, China, 28 a 31 de Marzo, (2010).

Field C.B., Barros V., Stocker T.F., and Dale Q., Managing the Risks of Extreme Events and Disasters to Advance Climate Change Adaptation Cambridge University Press, Cambridge, UK, (2012).

Fortier, C., Assani, A. L. I. A., and Mesfioui, M., Comparison of the interannual and interdecadal variability of heavy flood characteristics upstream and downstream from dams in inversed hydrologic regime: Case study of Matawin River. River Research and Applications, 27(1), 1277-1289, (2011).

Musgens, F., Quantifying market power in the German wholesale electricity market using dynamic multiregional dispatch model.Journal of Industrila Economics, 54(4), 471-489, (2006)

Guohua, L., Guoli, W., and Shufeng, X., A Multi-reservoir Flood Operating System Based on Fuzzy Messages. Proceedings of the Sixth International Conference on Fuzzy Systems and Knowledge Discovery, 22-28, Tianjin, China, 14 a 16 de Agosto (2009).

Hao, Y., Research on optimal operation of multipurpose reservoirs: Theory and application, Proceedings of the $4^{\text {th }}$ International Conference on Computer Science \& Education, 56-61, Nanning, China, 25 al 28 de Julio, (2009)

Jothiprakash, V., Shanthi, G., and Arunkumar, R., Development of Operational Policy for a Multi-reservoir System in India using Genetic Algorithm. Water Resources Management, 25(10), 2405-2423, (2011).

Leiva, Pablo., El medio ambiente en Colombia, Instituto de Hidrología, Meterología y Estudios Ambientales. IDEAM, Facultad de Ciencias. Universidad Nacional de Colombia, (2001).

Ma, C., Wang, H., and Lian, J., Short-term electricity dispatch optimization of Ertan hydropower plant based on data by field tests. Journal of Renewable and Sustainable Energy, 3(6), 127-138, (2011). 
Mahmud, M. R., and Hashim, M., Operational satellite-based watershed monitoring systems (SAWMOS) for large humid tropical catchment environment. IEEE Colloquium on Humanities, Science and Engineering, 687-691, Penang, Malasia, 5 a 6 de diciembre (2011).

Minville, Marie., Brissette, Francois., and Leconde, Robert., Impacts and Uncertainty of Climate Change on Water Resource Management of the Peribonka River System (Canada), Journal of Water Recourses, Planning and Management, 136(3), 376-385, (2010).

Ngo, L. L., Optimizing reservoir operation. A case study of the Hoa Binh reservoir, Vietnam. Tesis de Doctorado, Institute of Environment and Resourses, Technical University of Denmark, Denmark (2006).

Philander, S. G. H., El Niño and La Niña, Geophysical Fluid Dynamics Laboratory, Princeton University, Princeton NJ 08542, Journal of the Atmospheric sciences, 42(23), 2652-2662, (1985).

Ruiz, M., Bernal, G., \& Polanía, J., Influencia del río Sinú y el mar caribe en el sistema lagunar de Cispatá, Boletín de Investigaciones Marinas y Costeras 37(1), 29-49, (2008).

Terzaghi, K. Peck, R.B y Moretto O., Mecánica de suelos en la Ingeniería Práctica, 55-57. El Ateneo Editorial, Buenos Aires, Argentina, (1980)

Villada F, García E y J.D. Molina, Pronóstico del precio de la energía eléctrica usando redes neuro-difusas. Revista Inf. Tecnol. Cit: 22(6), 111-120, (2011).

Xu, C.-guang, and Zhao, M.-huan, Flood Control Compensation Benefit Calculation of Large Reservoir. Proceedings of the 4th International Conference on Bioinformatics and Biomedical Engineering, 1-4, Chengdu, China, 18 al 20 de Junio (2010). 
